\title{
Maternal peri-conceptional undernourishment perturbs offspring sperm methylome
}

\author{
Paola Toschi ${ }^{1, *}$, Emanuele Capra ${ }^{2,3, *}$, Debora A Anzalone ${ }^{1}$, Barbara Lazzari ${ }^{2,3}$, Federica Turri ${ }^{3}$, \\ Flavia Pizzi ${ }^{3}$, Pier A Scapolo ${ }^{1}$, Alessandra Stella ${ }^{3}$, John L Williams ${ }^{4}$, Paolo Ajmone Marsan² and \\ Pasqualino Loi $^{1}$ \\ ${ }^{1}$ Laboratory of Experimental Embryology, Faculty of Veterinary Medicine, University of Teramo, Teramo, Italy, \\ ${ }^{2}$ Department of Animal Science, Food and Technology - DIANA, and Nutrigenomics and Proteomics Research \\ Center - PRONUTRIGEN, Università Cattolica del Sacro Cuore, Piacenza, Italy, ${ }^{3}$ Institute of Agricultural Biology and \\ Biotechnology (IBBA), National Research Council (CNR), Lodi, Italy and ${ }^{4}$ Davies Research Centre, School of Animal \\ and Veterinary Sciences, University of Adelaide, Adelaide, South Australia, Australia
}

Correspondence should be addressed to P Toschi; Email: ptoschi@unite.it

*(P Toschi and E Capra contributed equally to this work)

\begin{abstract}
The genotype of an organism is stable throughout its life; however, its epigenome is dynamic and can be altered in response to environmental factors, such as diet. Inheritance of acquired epigenetic modifications by the next generation occurs through the germline, although the precise mechanisms remain to be elucidated. Here, we used a sheep model to evaluate if modification of the maternal diet (CTR; control, UND: undernutrition; FA: undernutrition and folic acid supplementation) during the peri-conceptional period affects the genome-wide methylation status of the gametes of male offspring. Sperm DNA methylation, measured by Reduced Representation Bisulfite Sequencing (RRBS), identified Differentially Methylated Regions (DMR) in offspring that experienced in utero undernutrition, both in UND (244) and FA (240), compared with CTR. Gene ontology (GO) analysis identified DMRs in categories related to sperm function, therefore we investigated whether the fertilizing capacity of the semen from the three groups differed in an in vitro fertilization assay. Spermatozoa from the undernourished groups showed lower motility and sperm chromatin structure abnormalities, represented by a higher percentage of DNA fragmentation and an increased number of immature cells, compared with CTR. While good quality blastocysts were obtained from all three groups, the proportion of embryos reaching the blastocyst stage was reduced in the UND vs CTR, an effect partially rescued by the FA treatment. The data reported here show that nutritional stress during early pregnancy leads to epigenetic modifications in the semen of the resulting offspring, the effects of which in next generation remain to be elucidated.

Reproduction (2020) 159 513-523
\end{abstract}

\section{Introduction}

The relationship between foetal exposure to malnutrition and onset of diseases in adult life has been hypothesized since the 1980s (Barker hypothesis; Barker et al. 1993). First described in human epidemiological studies on the effects of maternal undernutrition on progeny during the Dutch HungerWinter, the phenomenon has subsequently been demonstrated in several animal models (Langley \& Jackson 1994, Langley-Evans et al. 1998, Rae et al. 2002, Zambrano et al. 2005). Undernutrition or malnutrition during pregnancy not only affects the health of the exposed individuals and their offspring, but in some cases the phenotype induced by environmental stressors may be inherited across generations (Patti 2013). It has been proposed that transmission of such environmentally acquired phenotypes may be mediated by epigenetic modification, which is described as transgenerational epigenetic inheritance (Gluckman et al. 2007).

Studies of the molecular mechanisms underlying epigenetic inheritance have focused on three classes of potential epigenetic information carriers: cytosine methylation, chromatin state, and small RNAs (Rando 2012). Retained histone modifications and abnormal populations of small noncoding RNAs in sperm have been implicated in the transgenerational inheritance of epigenetically controlled phenotypes that vary following male exposure to stress or malnutrition (Rodgers et al. 2015, Siklenka et al. 2015). These epigenetic mechanisms of inheritance have been extensively studied in flies (Seong et al. 2011) and worms (Greer et al. 2010, Ashe et al. 2012). However, embryo development in these species is different from mammals. Cytosine methylation is the best characterized epigenetic 
mechanism regulating the expression of the mammalian genome, but it is less stable than previously thought, with dynamic changes during embryo development and gametogenesis (Hackett \& Surani 2013). Nevertheless, methylation of DNA appears to play a primary role in the transmission of specific phenotypes to the next generations via epigenetic modifications in the germline (Daxinger \& Whitelaw 2012, Radford et al. 2014, Wei et al. 2015, Huypens et al. 2016).

Nutritional stress experienced during early development alters DNA methylation patterns in the offspring via epigenetic changes in the gametes, although there is no clear link between the kind of stress and the specific epigenetic modification that result (Martínez et al. 2014, Radford et al. 2014). Most of the genome demethylation data has been obtained from mouse studies. Mouse primordial germ cells (PGCs) appear around day (E) 6.25 of embryonic development and undergo extensive DNA demethylation, which is essential for their transition from PGCs to gonocyte (12.5dpc) (Seisenberger et al. 2013, Hill et al. 2018). Following erasure, the genomic methylation patterns that are acquired in the germline differ markedly for male and female gametes, especially at imprinted loci that have allele-specific gene expression in the offspring. The second DNA demethylation wave takes place during early embryonic development and is crucial for establishing pluripotency (Hackett \& Surani 2013, Messerschmidt et al. 2014). It is, therefore, unclear how the epigenetic information is passed to the progeny. There is less information on the timing of epigenetic reprogramming in sheep PGCs, but at day 28 sheep PGCs are largely localized in the genital ridges, suggesting that by this time methylation marks have been mostly erased (Ledda et al. 2010, Van Soom et al. 2013). The genome epi-modification occurring during these two developmental phases are particularly sensitive to environmental factors (Faulk \& Dolinoy 2013). Exposure of a pregnant female to environmental insults during this crucial time-dependent developmental window can lead to alteration of the epigenetic profile of both PGC and the zygote (Bernal \& Jirtle 2010).

The present study was carried out in sheep, for which the nutritional requirements and the reproductive physiology are well characterized and that are commonly used as a model for human foetal development (Sinclair et al. 2010). We evaluated whether maternal diet restriction during early pregnancy affects methylation of spermatozoa and if this has developmental effects on embryos following in vitro fertilization. Furthermore, we investigated if folic acid, a B complex vitamin critical for one carbon (C1) metabolism that acts as methyl donor for DNA methylation (Cetin et al. 2010, Anderson et al. 2012), may counteract the effects of restricted nutrition during

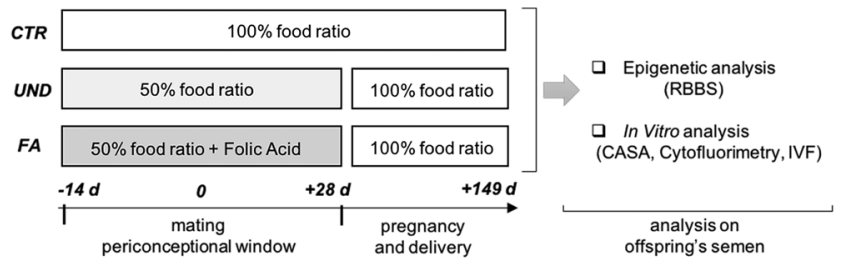

Figure 1 Schematic representation of the nutritional treatment of the three groups of sheep (CTR, UND, and FA) during the peri-

conceptional period $(-14 /+28$ days) in order to evaluate the effect on epigenetic patterns in the gametes of the male offspring.

the critical developmental window characterized by progressive DNA re-methylation.

\section{Materials and methods}

All experimental procedures involving animals were conducted in accordance with DPR 27/1/1992 (Animal Protection Regulations of Italy) in conformity with European Community regulation 86/609. All animal experiments were performed under the authority of the local ethical committee approval (CEISA-comitato etico interistituzionale per la sperimentazione animale No PROT.UNCHD12-222/2014). All chemicals were obtained from Sigma Aldrich unless otherwise stated. The experimental design of the study is shown in Fig. 1.

\section{Animal nutrition and breeding management}

In a commercial flock, 27 multiparous Sardinian sheep were randomly assigned into three groups of nine ewes and fed one of the three diets during the periconceptional period, from 14 days before mating (oocyte maturation) until 28 days after mating (early organogenesis), which corresponds to Carnegie stage 19 (Butler \& Juurlink 1987): 42 days in total.

Ewes in the control group (CTR) were fed ad libitum with a balanced diet satisfying nutritional requirements of pregnant ewes (300 g/animal/day). Ewes of the undernutrition group (UND) were fed a restricted diet (50\% of the requirements: 150 $\mathrm{g} /$ animal/day) of the same diet. Ewes in the folic acid group (FA) were fed the same as the UND group, but were supplemented with folic acid (15 mg; Folina; Teofarma) injected i.m. every $24 \mathrm{~h}$ for during the 42 days of the nutritional treatment. The nutritional stress imposed on the UND animals was sufficient to induce a gross caloric restriction without affecting the health and well-being. All animals had a full clinical assessment each week. The Table 1 provides the details of the nutritional regimes used in the three groups.

To optimise the pregnancy rate, oestrus was synchronized by treating all ewes with intravaginal progesterone sponges (25-mg Chronogest; Intervet) for 14 days. At the time of sponge removal, a ram of proven fertility was used to mate the three nutritional treatment groups, temporarily hosted in a common paddock. From the time of mating onwards, ewes were group housed in pens under natural day length conditions with ad libitum access to water. At day 28 of gestation, all ewes 
Table 1 Diet composition (complementary feed and pelleted alfalfa) in normal (100\%: $300 \mathrm{~g} / \mathrm{animal}$ ) and restricted (half daily ratio-50\%: 150 g/animal) experimental groups.

\begin{tabular}{lc}
\hline Ingredients & Amount $(\%)$ \\
\hline Complementary feed OV3 (\#05.0003; Europa mangimi; Italy) \\
$\quad$ Crude protein & 16.70 \\
Crude cellulose & 12.90 \\
Crude ashes & 8.80 \\
Crude oil and fats & 3.20 \\
$\quad$ Sodium & 0.20 \\
Pelleted Alfalfa (\#964; Mangimi Ariston srl; Italy) & \\
$\quad$ Crude Protein & 14 \\
Crude cellulose & 32 \\
Crude ashes & 11 \\
\hline
\end{tabular}

were returned to an ad libitum diet, to meet daily energy and nutritional needs and to ensure that all animals had regained sufficient fat reserves to maintain the pregnancy for the 149.7 days average gestation period for the Sarda sheep breed and for the onset and maintenance of lactation. Following parturition, all animals were conventionally managed, and all male lambs ( $n=11$; 4 CTR, 3 UND; 4 FA) were weighed at seven time points from birth to day 30 (day 0, 1, 2, 5, 10, 20, and 30). For subsequent analysis, we excluded lambs born from twin pregnancies in order to avoid any confounding factors. A nonparametric Kruskal-Wallis test was used to assess the effect of nutritional treatment on lamb body weight using Graph Pad Prism software. Data are presented as mean \pm S.E.M., with a $P$ value $<0.05$ indicating statistically significant differences between groups.

\section{Semen collection}

The ram lambs were trained for semen collection with an artificial vagina after puberty (around 9 months in Sarda breed). Immediately after collection, semen volume and motility was evaluated under a stereomicroscope and concentration was assessed using a Burker chamber. Part of each ejaculate was processed for molecular analysis and the rest cryopreserved.

\section{Methylome analysis}

\section{Sperm preparation for RRBS analysis}

After collection, the ejaculate was diluted in $3 \mathrm{~mL}$ of basic medium (BM), composed of $300 \mathrm{mM}$ TRIS base, $105 \mathrm{mM}$ citric acid, $82 \mathrm{mM}$ fructose, 150,000 IU penicillin G, $2 \mathrm{mM}$ streptomycin in $(67.20 \mathrm{~mL})$ double-distilled water $(\mathrm{ddH} 20)$ ( $\mathrm{pH}$ was adjusted to 6.7/6.8) and centrifuged at $461 \mathrm{~g}$ for 10 min to remove seminal plasma. Then, $1 \mathrm{~mL}$ of supernatant was discarded and replaced with $1 \mathrm{~mL}$ of clean BM, mixed and centrifuged again at $101 \mathrm{~g}$ for $5 \mathrm{~min}$. Next, tubes were gently placed in a humidified atmosphere at $38.5^{\circ} \mathrm{C}$ and $5 \% \mathrm{CO}_{2}$, angled at $45^{\circ}$, for 45 to $60 \mathrm{~min}$, to allow the motile spermatozoa to swim up. After incubation, supernatant was collected from the top of the liquid phase in $200 \mu \mathrm{L}$ aliquots. From each collection, $5 \mu \mathrm{L}$ were examined under the microscope, to exclude aliquots containing somatic cells. Once a minimum of $500 \mu \mathrm{L}$ of supernatant free of somatic cells had been collected, sperm concentration was assessed and tubes centrifuged at
$10,000 \mathrm{~g}$ for $10 \mathrm{~min}$. Finally, the pellet was resuspended at a concentration of $25 \times 10^{6}$ spermatozoa per $100 \mu \mathrm{L}$ in BM and stored at $-80^{\circ} \mathrm{C}$ until processed.

\section{Reduced Representational Bisulfite (RRBS) Library preparation and sequencing}

Genomic DNA from each sperm sample was isolated using the NucleoSpin ${ }^{\circledR}$ Tissue kit (Macherey-Nagel, Düren, Germany), following the manufacturer's instructions. DNA concentration was estimated by PicoGreen $₫$ (Thermo Fisher Scientific). One $\mu \mathrm{g}$ of DNA was digested with Mspl (New England Biolabs, UK) by overnight incubation at $37^{\circ} \mathrm{C}$, following the manufacturer's instruction. Libraries were generated using the TruSeq ${ }^{\circledR}$ DNA PCR-Free Library Preparation kit (Illumina). After adapter ligation, samples were converted using an EpiTect Bisulfite Kit (Qiagen) and finally PCR amplified with KAPA HiFi Uracil+ (Kapa Biosystems). RRBS libraries were sequenced on an Illumina Hiseq 3000 (San Diego, CA, United States) to generate 150-base paired-end reads.

\section{Methyl-seq library preparation and sequencing for RRBS validation}

For RRBS validation, methyl-seq libraries were prepared from genomic DNA isolated from each sperm sample. DNA was sonicated to produce fragments of about $350 \mathrm{bp}$. MethylMinerTM kit (Invitrogen) was used to enrich highly methylated DNA fragments in the sample. The enriched methylated fragments were then converted to libraries with TruSeq ${ }^{\circledR}$ Nano Library Preparation Kit (Illumina, San Diego, CA, USA). Methyl-seq libraries were sequenced on an Illumina Hiseq 3000 (San Diego, CA, United States) to generate 150base paired-end reads.

\section{Bioinformatic analysis}

The preliminary quality control of raw sequence reads was carried out with FastQC (http://www.bioinformatics.babraham. ac.uk/projects/fastqc/). Quality control and removal of adapters from the Illumina raw sequences was performed with Trim Galore (https://www.bioinformatics.babraham.ac.uk/projects/ trim_galore/). Data are available in the Sequence Reads Archive (SRA), BioProject accession number, PRJNA491660.

Bismark software v.0.17.0 was used to align each read to a bisulfite-converted sheep genome (Oar_v3.1) with option $-\mathrm{N}$ 1. Methylation calls were extracted using the Bismark methylation_extractor function. Seqmonk software (version 1.36.0) (http://www.bioinformatics.babraham.ac.uk/projects/ seqmonk/) was used for visualization and analysis of the Bismark output. To detect regions having sufficient sequence coverage, the reference genome was split into 400-bp sliding windows with a 200-bp offset, and methylation percentage was calculated for each window containing at least six cytosine with $5 \times$ coverage. Only Methylated Regions (MRs) with sufficient sequence coverage in all nine samples were considered and analysed further. Differentially methylated regions (DMRs) were identified by comparing CTR vs UND, CTR vs FA, and UND vs FA. DMRs were calculated using the logistic regression filter in $\mathrm{R}(\mathrm{FDR}<0.05$, absolute cut-off of 
20\%) (Ziller et al. 2015). Hierarchical analyses in Genesis software (Sturn et al. 2002) were used to illustrate sample clustering. To compute shared DMRs and DMR associated genes across comparisons, commonality analysis was run and visualized through Venn diagrams, using the Venn Diagrams software (http://bioinformatics.psb.ugent.be/webtools/Venn/). DMR associated genes were classified according to classical GO categories, using the Cytoscape plug-in ClueGO which integrates GO (Bindea et al. 2009) and enhances biological interpretation of large lists of genes. In ClueGO, the $P$ value was calculated with the Fisher Exact Test corrected using the Bonferroni step down method.

For validation, after quality assessment with FastQC, methyl-seq raw sequences were trimmed with Trimmomatic and subsequently mapped to sheep reference genome (Oar_v3.1). BAM files were imported into Seqmonk software (version 1.36.0) and DMRs wee quantitated by calculating the normalized coverage for each region.

\section{In vitro semen analysis}

\section{Sperm cryopreservation}

Sperm was frozen as previously described (Anzalone et al. 2016). Briefly, Basic Medium (BM, see previous section for composition) used for the preparation of two other media, Medium A $(67.20 \%$ of $\mathrm{BM}+20 \%$ of egg yolk $+12.8 \%$ of $\mathrm{ddH} 2 \mathrm{O})$ and Medium B $(67.20 \% \quad \mathrm{BM}+20 \%$ of egg yolk $+12.80 \%$ of glycerol). Medium A and Medium B were added in equal volumes to the ejaculate to reach a final concentration of $400 \times 10^{6}$ spermatozoa $/ \mathrm{mL}$. Medium A was added to the ejaculate first and transferred to $4^{\circ} \mathrm{C}$ and cooled for $2 \mathrm{~h}$ at a controlled rate $\left(\sim-0.2^{\circ} \mathrm{C} / \mathrm{min}\right)$. Medium $\mathrm{B}$ was then added to the suspension and left for further $2 \mathrm{~h}$ at $4^{\circ} \mathrm{C}$. Next, $250 \mu \mathrm{L}$ straws were filled, sealed with polyvinyl alcohol (PVA) and placed onto a metallic grid, and stabilized at $4{ }^{\circ} \mathrm{C}$ for $2 \mathrm{~h}$. Finally, the straws were exposed to liquid nitrogen (LN) vapour $\left(-80^{\circ} \mathrm{C}\right)$ in a Dewar flask for 6 min before being plunged into $\mathrm{LN}$, where they were stored until use.

\section{Computer-assisted semen analysis (CASA)}

Sperm kinetic parameters were assessed using a CASA (Computer-Assisted Semen Analysis) system (ISAS® v1, Spain) as described in previous work (Capra et al. 2017). Samples were diluted to $\sim 30 \times 10^{6}$ cells $/ \mathrm{mL}$ with EasyBuffer $B \circledR(I M V$ Technologies, L'Aigle, France). A pre-warmed $\left(37^{\circ} \mathrm{C}\right)$ Makler counting chamber $(10 \mu \mathrm{m}$ depth) was loaded with $10 \mu \mathrm{L}$ of each sample, and at least three fields were acquired. Two technical replicates per sample were performed. Using a $10 \times$ objective in phase contrast, the image was relayed, digitized, and analyzed by the ISAS ${ }^{\circ}$ software with user-defined settings as follows: frames acquired, 25; frame rate, $20 \mathrm{~Hz}$;

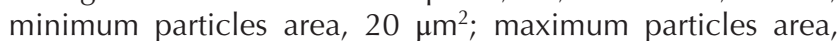
$70 \mu \mathrm{m}^{2}$; and progressivity of the threshold straightness, $70 \%$. Spermatozoa speed was assigned to three broad categories: rapid $(50 \mu \mathrm{m} / \mathrm{s})$, medium $(25 \mu \mathrm{m} / \mathrm{s})$, and slow $(10 \mu \mathrm{m} / \mathrm{s})$. CASA kinetics parameters were follows: total motility (MOT TOT, \%), progressive motility (PRG, \%), curvilinear velocity $(\mathrm{VCL}, \mu \mathrm{m} / \mathrm{s})$, straight-line velocity $(\mathrm{VSL}, \mu \mathrm{m} / \mathrm{s})$, average path velocity (VAP, $\mu \mathrm{m} / \mathrm{s}$ ), linearity coefficient (LIN, \% =VSL/VCL $\times 100)$, amplitude of lateral head displacement $(\mathrm{ALH}, \mu \mathrm{m})$, straightness coefficient $(\mathrm{STR}, \%=\mathrm{VSL} / \mathrm{VAP} \times 100)$, wobble coefficient $(\mathrm{WOB}, \%=\mathrm{VAP} /$ $\mathrm{VCL} \times 100)$, and beat cross frequency $(\mathrm{BCF}, \mathrm{Hz})$.

\section{Flow cytometry analysis}

Measurements were recorded using a Guava easyCyteTM 5HT microcapillary flow cytometer with cytoSoft and IMV easySoft software (Merck KGaA, Darmstadt, Germany; distributed by IMV Technologies). The fluorescent probes were excited by a $20 \mathrm{~mW}$ argon ion laser (488 nm). Forward Scatter (FSC) v. side-scatter plots were used to separate sperm cells from debris. Non-sperm events were excluded from further analysis. Fluorescence detection was set with three photomultiplier tubes: detector FL-1 (green: 525/30 nm), detector FL-2 (yellow/ orange: 583/26 nm), and detector FL-3 (red: 655/50 nm). A total of 5000 sperm events per sample were analyzed at a flow rate 200 cells/s.

Nuclear chromatin was analyzed as previously described (Gliozzi et al. 2017) using acridine orange, a fluorochrome that turns from red to green depending on the degree of chromatin compaction.

The technique is based on the susceptibility of sperm DNA to acid-induced denaturation, as low-pH treatment causes partial DNA denaturation in sperm with altered chromatin structure. Acridine orange is a planar molecule which intercalates into dsDNA but stacks on ssDNA causing a metachromatic shift from green (dsDNA) to red fluorescence (ssDNA), when exposed to the $488 \mathrm{~nm}$ laser of the flow cytometer.

The assessment was performed using the sperm chromatin structure assay (Evenson \& Jost 2000): $3.0 \times 10^{5}$ cells were diluted in $200 \mu \mathrm{L}$ of TNE buffer (0.01 M Tris-HCl, $0.15 \mathrm{M} \mathrm{NaCl}$, $1 \mathrm{mM}$ ethylenediaminetetraacetic acid (EDTA), $\mathrm{pH} 7.4$ ) and added to $400 \mu \mathrm{L}$ of an acidic solution (Triton X-100 0.1\%, 0.15 $\mathrm{M} \mathrm{NaCl}, 0.08 \mathrm{M} \mathrm{HCl} ; \mathrm{pH} 1.2)$. After $30 \mathrm{~s}$, cells were stained with $1.2 \mathrm{~mL}$ of acridine orange solution $(6 \mu \mathrm{g} / \mathrm{mL}$ in $0.1 \mathrm{M}$ citric acid, $0.2 \mathrm{M} \mathrm{Na}^{2} \mathrm{HPO}^{4}, 1 \mathrm{mM}$ EDTA, $0.15 \mathrm{M} \mathrm{NaCl}$; pH 6). After 2.5 min, two replicates per sample were read on a flow cytometer (Guava easyCyteTM 5HT microcapillary flow cytometer). The fluorescent probes were excited by a $20 \mathrm{~mW}$ argon ion laser $(488 \mathrm{~nm})$. Forward scatter (FSC) v. side-scatter plots were used to separate sperm cells from debris. Non-sperm events were excluded from further analysis. Fluorescence detection was set with three photomultiplier tubes: detector FL-1 (green: 525/30 $\mathrm{nm}$ ), detector FL-2 (yellow/orange: 583/26 nm), and detector FL-3 (red: 655/50 nm). Calibration was carried out using standard beads (Guava ${ }^{\circledR}$ Easy Check Kit; Merck Millipore). Data were analyzed using cytoSoft and IMV easySoft software (Merck KGaA, Darmstadt, Germany; distributed by IMV Technologies), with the following parameters: Alpha-T that is indicative of the shift from green to red fluorescence and is expressed as the ratio of red to total fluorescence intensity $(\mathrm{red} /($ red + green $))$, which quantifies the degree of abnormal chromatin structure that has an increased susceptibility to acid-induced denaturation; S.D. of Alpha-T (ATSD) that shows the extent of abnormality in chromatin structure within a population; \%DFI that indicates the percentage of sperm with fragmented DNA; and percentage of sperm with high green $(\% \mathrm{HG})$ fluorescence that is representative of the percentage 
of immature cells with reduced nuclear condensation (incomplete histone-protamine exchange).

\section{Statistical analysis}

Data obtained from CASA and flow cytometry measurements were analysed using the SAS TM package v 9.4 (SAS Institute Inc., Cary, NC, USA). The General Linear Model procedure (PROC GLM) was used to evaluate the effect of the experimental groups (CTR, UND, FA) on semen quality parameters. Results are given as adjusted least squares means $($ LSM \pm IS.E.M.

\section{In vitro sheep embryo production}

\section{Oocyte collection and in vitro maturation (IVM)}

Sheep oocytes were retrieved from ovaries collected from a local slaughterhouse. Oocytes were aspirated using $21 \mathrm{~g}$ needles into warm TCM-199 medium (Gibco, Life Technologies) buffered with $0.47 \%$ HEPES buffer and supplemented with $0.005 \%$ (w:v) heparin. Only oocytes with 2-3 layers of compact cumulus cells were selected for IVM and incubated for $24 \mathrm{~h}$ in a humidified atmosphere at $38.5^{\circ} \mathrm{C}$ and $5 \% \mathrm{CO}_{2}$ in IVM medium. The latter was composed of bicarbonate-buffered TCM-199 (Gibco) containing 2 mM glutamine, 0.3 mM sodium pyruvate, $100 \mu \mathrm{M}$ cysteamine, $10 \%$ foetal bovine serum (FBS) (Gibco), $5 \mu \mathrm{g} / \mathrm{mL}$ follicle stimulating hormone (FSH) (Ovagen, ICP, Auckland, New Zealand), $5 \mu \mathrm{g} / \mathrm{mL}$ luteinizing hormone (LH), and $1 \mu \mathrm{g} / \mathrm{mL} \beta$-estradiol. Following IVM, mature oocytes (MII) showed expanded cumulus and extruded first polar body.

\section{In vitro fertilization (IVF)}

In vitro fertilization was performed as previously described (Anzalone et al. 2016). Briefly, frozen semen was fast-thawed in a waterbath at $35^{\circ} \mathrm{C}$ for $10 \mathrm{~s}$ and centrifuged in SOFcontaining $0.4 \%$ BSA (w/v), at $180 \mathrm{~g}$ for $5 \mathrm{~min}$. Matured oocytes were gently pipetted in $300 \mathrm{U} / \mathrm{mL}$ hyaluronidase solution (dissolved in H199) to partially remove granulosa cell, then placed into $50 \mu \mathrm{L}$ drops of IVF medium covered by mineral oil, and incubated with sperm $\left(5 \times 10^{6}\right.$ spermatozoa/ $\mathrm{mL}$ ) overnight, in a humidified atmosphere at $38.5^{\circ} \mathrm{C}, 5 \%$ $\mathrm{CO}_{2}$, and $7 \% \mathrm{O}_{2}$. The day after, putative zygotes were pipetted in SOF-medium to remove most of the spermatozoa attached to the zona pellucida and cultured as described.

\section{Embryo culture}

All putative zygotes from in vitro fertilized oocytes were cultured into $20-\mu \mathrm{L}$ drops of SOF-enriched with $2 \%$ (v:v) basal medium Eagle essential amino acids (EAA), 1\% (v:v) minimum essential medium (MEM)-nonessential amino acids (NEAA) (Gibco), $1 \mathrm{mM}$ glutamine, and $8 \mathrm{mg} / \mathrm{mL}$ fatty acid-free BSA, covered by mineral oil. The medium was renewed on day 3 (SOF supplemented with $0.27 \mathrm{mg} / \mathrm{mL}$ glucose (SOF+), $2 \%$ EAA, $1 \%$ NEAA), on day 5 (SOF+ with $10 \%$ of charcoal stripped FBS (cs-FBS), 2\% EAA, 1\% NEAA), and on day 6 (1:1 MEM/M199 enriched with 10\% cs-FBS, $2.5 \mu \mathrm{g} / \mathrm{mL}$ gentamicin and $1 \%$ sodium pyruvate), and then incubated until day $7-8$. The in vitro development was evaluated on day 1 (2 cell-stage) and the $7 / 8$ th day (blastocyst stage). Fisher's exact test was used to compare in vitro embryo developmental stages between the groups. Data were analyzed using PRISM software (version 6). GraphPad and the values were considered to be significantly different when $P<0.05$.

\section{Results}

\section{Lamb body weight}

Body weight of male lambs from controls (CTR) and the two feed restricted groups (UND and FA) showed no significant difference (Fig. 2) at birth (CTR: $3.53 \pm 0.50$ kg; UND: $3.96 \pm 0.28 \mathrm{~kg} ; \mathrm{FA}: 4.20 \pm 0.35 \mathrm{~kg})$ and up to day 30 post-partum $(7.90 \pm 0.70 \mathrm{~kg} ;$ UND: $10.30 \pm 0.37$ kg; FA: $9.70 \pm 1.90 \mathrm{~kg}$ ).

\section{Sperm methylation}

The average number of sequence reads per sperm sample was 28.1 M (ranging from $10.9 \mathrm{M}$ to 56.6 $M)$. Reduced representation bisulphite sequencing (RRBS) showed a high-level cytosine methylation in the context of CpG, which was, on average, $67.3 \%$ across all sperm samples (Supplementary file 1, see section on supplementary materials given at the end of this article). A total of 86,944 MRs were detected across all samples by SeqMonk software. Considering differential cytosine methylation, where variation was greater than $20 \%$, the pairwise comparison of sperm methylation between experimental groups identified 244 differentially methylated regions (DMRs) in CTR vs UND, 240 DMRs in CTR vs FA, and 106 DMRs in UND vs FA. Annotation of the 244, 240, and 106 DMRs identified 75, 69, and 38 DMR associated genes (Supplementary file 2). The number of DMRs from the comparison of offspring from undernourished mothers (UND) with undernourished mothers supplemented with folic acid (FA) was considerably lower than that

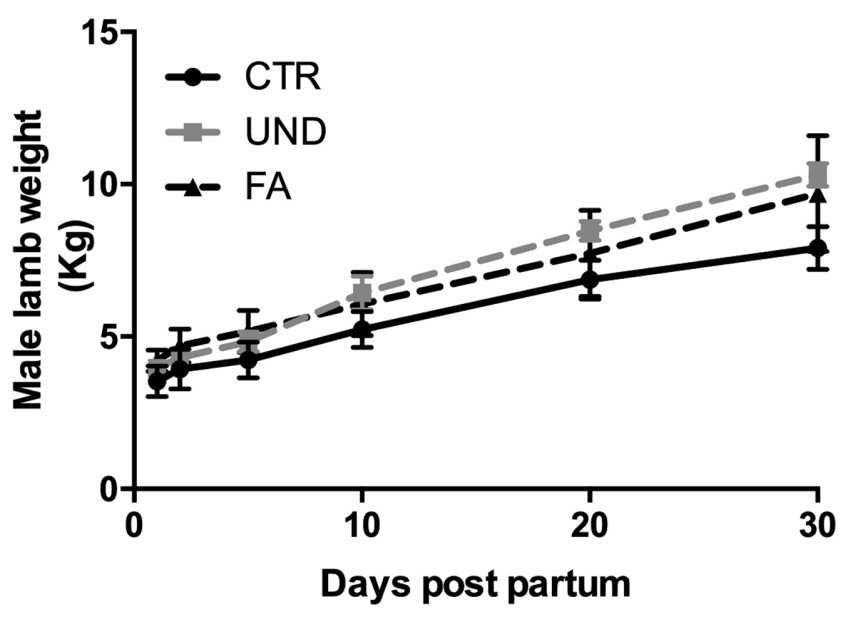

Figure 2 Weights of male lamb for the three groups of sheep. Animals were weighed at birth and at five subsequent time points (day 2, 5 , 10, 20, and 30) in both treated (UND and FA) and CTR groups. 
observed in the other two comparisons (CTR vs UND and CTR vs FA). DMR associated genes shared by CTR vs UND and CTR vs FA comparisons were $19.7 \%$ (87 out of 442 ) and $22.3 \%$ (29 out of 130), respectively, of the total DMRs and DMR associated genes (Fig. 3). Hierarchical clustering based on DMRs obtained from different contrasts (CTR vs UND, CTR vs FA, and UND vs FA) highlighted some common patterns. In particular, all CTR animals consistently clustered together, as did UND1 and UND2. UND3 consistently clustered with FA3 and behaved as belonging to the FA rather than to the UND group. CTR vs UND DMRs separated controls from underfed animals, with the exception of FA2 that clustered with the CTR group (Fig. 4A). CTR vs FA DMRs were even more effective in distinguishing underfed from CTR animals (Fig. 4B). Both sets of DMRs clustered undernourished animals receiving or not receiving FA supplementation separately (with exception of UND3). UND vs FA DMRs showed a closer relationship between CTR and FA compared to CTR and UND (with the exception of UND3), suggesting a possible role of FA supplementation in restoring the epigenetic asset (Fig. 4C).

GO analysis was performed on the GO classes that included at least two differentially methylated genes. DMR associated genes between CTR vs UND were involved in the regulation of I-Kappa $B(I-K B)$ and NF-kappa B (NF-KB) signalling, regulation of chondroitin sulphate synthesis, potassium ion import, and response to parathyroid hormone. Whereas the CTR vs FA comparison identified genes within DMR related to biotin metabolism, response to forskolin, and regulation of mitochondrial function. Finally, comparing UND vs FA identified variation in the methylation of genes associated to neural development, response to glucagon, ISG15-protein conjugation, and activation of adenylate cyclase activity (Table 2 ).
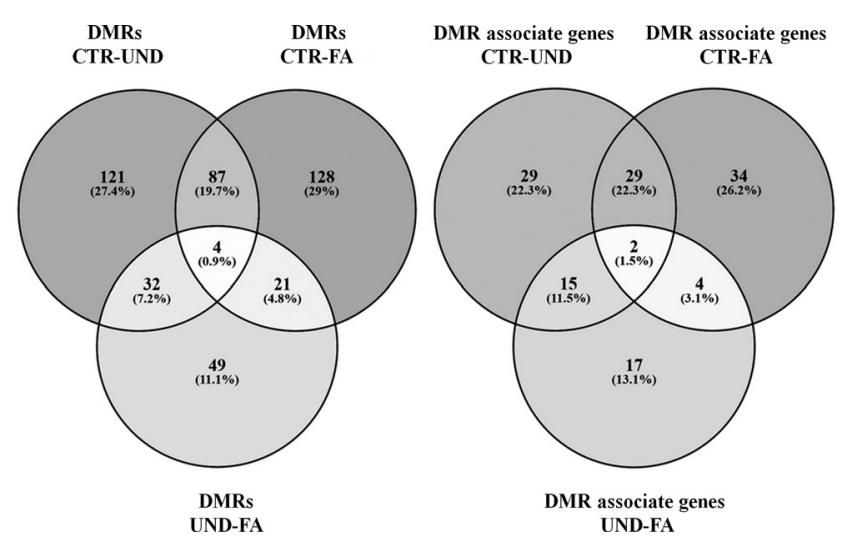

Figure 3 Venn diagrams representing the number and percentage of differentially methylated regions (DMRs) and DMR associated genes shared and unique in comparisons of CTR vs UND, CTR vs FA, and UND vs FA groups.
A

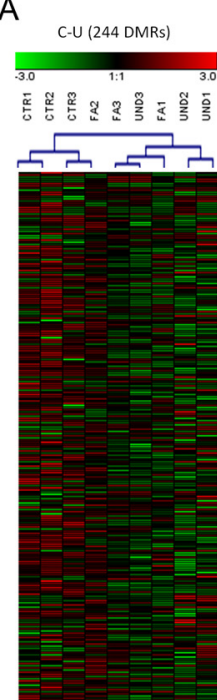

B

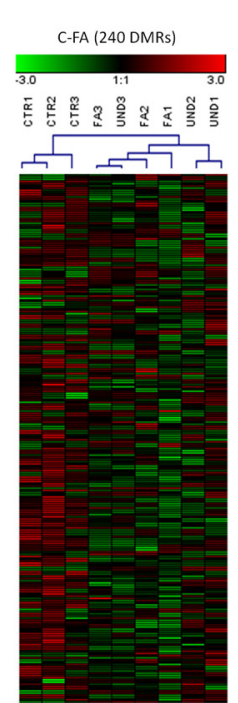

C

U-FA (106 DMRS)

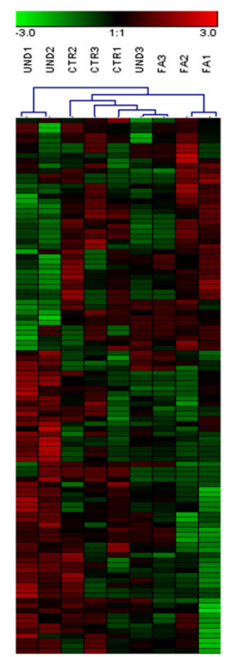

Figure 4 The three heatmaps comparing the methylation status of sheep at DMRs detected in the three comparisons: (A) 244 differentially methylated regions (DMRs) in CTR vs UND, (B) 240 DMRs in CTR vs FA, and (C) 106 DMRs in UND vs FA. The list of DMRs is only partially overlapping across comparisons.

The 244, 240, and 106 DMRs found in CTR vs UND, CTR vs FA, and UND vs FA, respectively, were verified by comparing the average RRBS methylation percentage and the average of normalized count obtained by methylseq. The intersect between the subsets representing the 25th percentile of the HMRs for each condition (CTR, UND, and FA) obtained by RRBS and methyl-seq showed a higher percentage of shared regions than that due to chance (Supplementary file 3).

\section{In vitro semen analysis}

Computer-assisted semen analysis (CASA) sperm kinetic parameters, including total motility (MOT TOT) and progressive (PRG) motility, were significantly lower in spermatozoa collected from both undernourished groups (UND and FA) compared with controls (Table 3).

Differences were also observed in the extent of chromatin structure abnormality, with a significant increase of Alpha-T S.D. (ATSD) for the FA groups compared with CTR, although not in the UND group with respect to the CTR group (Table 3).

\section{Embryo development}

Following in vitro fertilization, significant differences in embryo development were seen between all groups (Table 4). At $24 \mathrm{~h}$, the percentage of two cells embryos was $31.5 \%$ in CTR, $23.6 \%$ in UND (44/186), and $39 \%(46 / 118)$ in the FA, and these differences are all statistically significant $(P=0.006615)$. The rate of embryos reaching blastocyst stage was $14.6 \%(26 / 178)$ 
Table 2 Gene Ontology (GO) analysis of DMR associated genes obtained by comparing CTR vs UND, CTR vs FA and UND vs FA groups.

\begin{tabular}{|c|c|c|c|c|}
\hline GOID & Associated Genes Found & GOTerm & $P$ Value & Corrected $\boldsymbol{P}$ value* \\
\hline \multicolumn{5}{|l|}{ CTR-UND } \\
\hline 1903659 & IL10, IL11 & regulation of complement-dependent cytotoxicity & $1.4 \mathrm{E}-04$ & $2.5 \mathrm{E}-03$ \\
\hline 0071374 & HDAC4, PRKCA & cellular response to parathyroid hormone stimulus & 2.7E-04 & $4.3 \mathrm{E}-03$ \\
\hline 0030397 & $\angle M N A, P R K C A$ & membrane disassembly & $1.1 \mathrm{E}-02$ & $1.1 \mathrm{E}-02$ \\
\hline 0051081 & LMNA, PRKCA & nuclear envelope disassembly & $1.1 \mathrm{E}-02$ & $1.1 \mathrm{E}-02$ \\
\hline 0043124 & FBXW11, IL10, TNIP1 & negative regulation of I-kappaB kinase/NF-kappaB signalling & $8.9 \mathrm{E}-04$ & $1.2 \mathrm{E}-02$ \\
\hline 0071107 & HDAC4, PRKCA & response to parathyroid hormone & 8.7E-04 & $1.3 \mathrm{E}-02$ \\
\hline 2000810 & $I K B K B, P R K C A$ & regulation of bicellular tight junction assembly & $1.4 \mathrm{E}-03$ & $1.9 \mathrm{E}-02$ \\
\hline 0072577 & IL10, IL11 & endothelial cell apoptotic process & 1.0E-02 & $2.1 \mathrm{E}-02$ \\
\hline 0010882 & $H D A C 4, P R K C A$ & $\begin{array}{l}\text { regulation of cardiac muscle contraction by calcium } \\
\text { ion signalling }\end{array}$ & $2.2 \mathrm{E}-03$ & $2.6 \mathrm{E}-02$ \\
\hline 0030206 & NCAN, XYLT1 & chondroitin sulphate biosynthetic process & $2.6 \mathrm{E}-03$ & $2.8 \mathrm{E}-02$ \\
\hline 0042347 & FBXW11, IL10 & negative regulation of NF-kappaB import into nucleus & $2.6 \mathrm{E}-03$ & $2.8 \mathrm{E}-02$ \\
\hline 0042345 & FBXW11, IL10 & regulation of NF-kappaB import into nucleus & $1.0 \mathrm{E}-02$ & $3.0 \mathrm{E}-02$ \\
\hline 0042348 & FBXW11, IL10 & NF-kappaB import into nucleus & $1.0 \mathrm{E}-02$ & $3.0 \mathrm{E}-02$ \\
\hline 0007077 & $\angle M N A, P R K C A$ & mitotic nuclear envelope disassembly & $9.2 \mathrm{E}-03$ & 3.7E-02 \\
\hline 0042992 & FBXW11, IL10 & negative regulation of transcription factor import into nucleus & $9.2 \mathrm{E}-03$ & 3.7E-02 \\
\hline 0050650 & NCAN, XYLT1 & chondroitin sulphate proteoglycan biosynthetic process & 3.7E-03 & 3.7E-02 \\
\hline 2000352 & IL $10, I L 11$ & negative regulation of endothelial cell apoptotic process & 3.7E-03 & 3.7E-02 \\
\hline 0010107 & KCNJ12, KCNJ6 & potassium ion import & 4.5E-03 & 4.1E-02 \\
\hline 2000351 & IL10, IL11 & regulation of endothelial cell apoptotic process & $8.5 \mathrm{E}-03$ & 4.2E-02 \\
\hline 0050654 & NCAN, XYLT1 & chondroitin sulphate proteoglycan metabolic process & $8.1 \mathrm{E}-03$ & $4.9 \mathrm{E}-02$ \\
\hline 1904036 & IL $10, I L 11$ & negative regulation of epithelial cell apoptotic process & $8.1 \mathrm{E}-03$ & 4.9E-02 \\
\hline \multicolumn{5}{|c|}{ 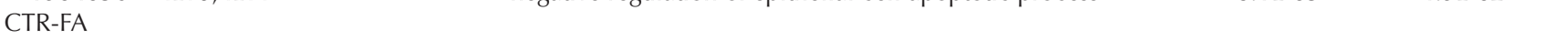 } \\
\hline 1904321 & ADCY5, EFNA5 & response to forskolin & 2.7E-04 & 2.7E-03 \\
\hline 1904322 & ADCY5, EFNA5 & cellular response to forskolin & 2.7E-04 & 2.7E-03 \\
\hline 0046928 & CACNA1A, PARK2, SEPT9 & regulation of neurotransmitter secretion & $4.9 \mathrm{E}-04$ & $4.4 \mathrm{E}-03$ \\
\hline 0050885 & ADCY5, CACNA1A, RBFOX1 & neuromuscular process controlling balance & $6.6 \mathrm{E}-04$ & $5.3 \mathrm{E}-03$ \\
\hline 0051588 & CACNA1A, PARK2, SEPT9 & regulation of neurotransmitter transport & $9.1 \mathrm{E}-04$ & $6.4 \mathrm{E}-03$ \\
\hline 0006768 & HLCS, PCCA & biotin metabolic process & $1.1 \mathrm{E}-03$ & $6.9 \mathrm{E}-03$ \\
\hline 0010823 & LMNA, PARK2 & negative regulation of mitochondrion organization & 8.6E-03 & $8.6 \mathrm{E}-03$ \\
\hline 0090201 & LMNA, PARK2 & $\begin{array}{l}\text { negative regulation of release of cytochrome c } \\
\text { from mitochondria }\end{array}$ & $1.9 \mathrm{E}-03$ & $9.4 \mathrm{E}-03$ \\
\hline 0043525 & HDAC4, PARK2 & positive regulation of neuron apoptotic process & 8.3E-03 & 1.7E-02 \\
\hline 0044003 & PARK2, PCCA & modification by symbiont of host morphology or physiology & 8.3E-03 & $1.7 \mathrm{E}-02$ \\
\hline 0045071 & $M X 1, P A R K 2$ & negative regulation of viral genome replication & 8.3E-03 & 1.7E-02 \\
\hline 1902803 & PARK2, SEPT9 & regulation of synaptic vesicle transport & $5.6 \mathrm{E}-03$ & $2.2 \mathrm{E}-02$ \\
\hline 0043113 & CACNA1A, DLG2 & receptor clustering & 8.0E-03 & $2.4 \mathrm{E}-02$ \\
\hline \multicolumn{5}{|l|}{ UND-FA } \\
\hline 0021631 & EPHB2, GLI3 & optic nerve morphogenesis & $3.6 \mathrm{E}-05$ & 5.0E-04 \\
\hline 0032020 & $U B E 2 E 2, U B E 2 L 6$ & ISG15-protein conjugation & $3.6 \mathrm{E}-05$ & $5.0 \mathrm{E}-04$ \\
\hline 0043535 & AMOTL1, MAP2K5, PRKCA & regulation of blood vessel endothelial cell migration & $8.2 \mathrm{E}-05$ & $1.1 \mathrm{E}-03$ \\
\hline 0021554 & $E P H B 2, G L I 3$ & optic nerve development & $1.8 \mathrm{E}-04$ & $2.2 \mathrm{E}-03$ \\
\hline 0032330 & GLI3, RARB & regulation of chondrocyte differentiation & $2.5 \mathrm{E}-03$ & $2.5 \mathrm{E}-03$ \\
\hline 0060831 & GLI3, PRKCA & $\begin{array}{l}\text { smoothened signalling pathway involved in dorsal/ventral } \\
\text { neural tube patterning }\end{array}$ & $2.5 \mathrm{E}-04$ & 2.7E-03 \\
\hline 0021532 & GLI3, PRKCA & neural tube patterning & $2.2 \mathrm{E}-03$ & 4.3E-03 \\
\hline 0071377 & ADCY5, PRKCA & cellular response to glucagon stimulus & $2.2 \mathrm{E}-03$ & 4.3E-03 \\
\hline 0007190 & ADCY5, PRKCA & activation of adenylate cyclase activity & $1.8 \mathrm{E}-03$ & $5.4 \mathrm{E}-03$ \\
\hline 0021544 & $G L I 3, R A R B$ & subpallium development & $5.4 \mathrm{E}-04$ & $5.4 \mathrm{E}-03$ \\
\hline 0021602 & EPHB2, GLI3 & cranial nerve morphogenesis & $8.8 \mathrm{E}-04$ & $6.2 \mathrm{E}-03$ \\
\hline 0031641 & $R A R B, T G$ & regulation of myelination & 1.1E-03 & $6.5 \mathrm{E}-03$ \\
\hline 0021904 & GLI3, PRKCA & dorsal/ventral neural tube patterning & $8.2 \mathrm{E}-04$ & $6.5 \mathrm{E}-03$ \\
\hline 0043536 & AMOTL1, PRKCA & positive regulation of blood vessel endothelial cell migration & 7.6E-04 & $6.8 \mathrm{E}-03$ \\
\hline 0045879 & GLI3, PRKCA & negative regulation of smoothened signalling pathway & 7.6E-04 & $6.8 \mathrm{E}-03$ \\
\hline 0003091 & $A D C Y 5, P R K C A$ & renal water homeostasis & 1.7E-03 & $6.8 \mathrm{E}-03$ \\
\hline 0007212 & ADCY5, RGS9 & dopamine receptor signalling pathway & 1.7E-03 & $6.8 \mathrm{E}-03$ \\
\hline 0048566 & GLI3, RARB & embryonic digestive tract development & $1.4 \mathrm{E}-03$ & $6.9 \mathrm{E}-03$ \\
\hline
\end{tabular}

$* P$ values were corrected with Bonferroni step down. 
Table 3 Computer-assisted semen analysis and flow cytometry recorded semen quality parameters in the control (CTR) and two undernourished (UND and FA) groups. Results are given as adjusted least squares means (LSM) \pm S.E.M.

\begin{tabular}{|c|c|c|c|}
\hline Parameters & CTR (LSM士S.E.M.) & UND (LSM \pm S.E.M.) & FA (LSM \pm S.E.M.) \\
\hline \multicolumn{4}{|l|}{ CASA } \\
\hline МOT TOT (\%)* & $72.6 \pm 3.68^{a}$ & $57.12 \pm 3.68^{a}$ & $54.75 \pm 3.00^{b}$ \\
\hline PRG $(\%)^{*}$ & $17.35 \pm 1.49^{a}$ & $12.56 \pm 1.49^{a b}$ & $11.22 \pm 1.21^{b}$ \\
\hline VCL $(\mu \mathrm{m} / \mathrm{s})$ & $26.57 \pm 5.17$ & $29.08 \pm 5.17$ & $21.96 \pm 4.22$ \\
\hline VSL $(\mu \mathrm{m} / \mathrm{s})$ & $77.41 \pm 3.50$ & $72.82 \pm 3.50$ & $69.66 \pm 2.86$ \\
\hline $\operatorname{VAP}(\mu \mathrm{m} / \mathrm{s})$ & $40.17 \pm 3.60$ & $41.27 \pm 3.60$ & $33.85 \pm 2.94$ \\
\hline LIN (\%) & $34.32 \pm 9.02$ & $40.73 \pm 9.02$ & $32.10 \pm 7.36$ \\
\hline STR $(\%)$ & $66.15 \pm 7.23$ & $69.13 \pm 7.23$ & $64.10 \pm 5.90$ \\
\hline WOB (\%) & $51.88 \pm 7.46$ & $57.31 \pm 7.46$ & $49.01 \pm 6.09$ \\
\hline $\mathrm{ALH}(\mu \mathrm{m})$ & $3.69 \pm 0.26$ & $3.42 \pm 0.26$ & $3.39 \pm 0.21$ \\
\hline $\mathrm{BCF}(\mathrm{Hz})$ & $5.35 \pm 0.94$ & $5.83 \pm 0.94$ & $4.34 \pm 0.76$ \\
\hline \multicolumn{4}{|l|}{ Cytofluorimetric } \\
\hline ALPHA-T & $0.4095 \pm 0.0027$ & $0.4085 \pm 0.0027$ & $0.4085 \pm 0.0022$ \\
\hline ATSD* & $0.0122 \pm 0.0002^{a}$ & $0.0127 \pm 0.0002^{\mathrm{a}}$ & $0.0138 \pm 0.0001^{b}$ \\
\hline DFI (\%) & $2.1625 \pm 0.2004$ & $2.5275 \pm 0.2004$ & $2.565 \pm 0.1636$ \\
\hline $\mathrm{HG}(\%)$ & $3.2825 \pm 0.4374$ & $2.165 \pm 0.4374$ & $2.8816 \pm 0.3571$ \\
\hline
\end{tabular}

ALH, amplitude of lateral head displacement; ALPHA-T, red/ (red+ green) fluorescence intensity; ATSD, Alpha-T standard deviation; BCF, beat cross frequency; DFI, fragmented DNA sperm; HG, high green fluorescence sperm; LIN, linearity coefficient (calculated as VSL/VCL x 100); MOT TOT, total motility; PRG, progressive motility; VAP, average path velocity; VCL, curvilinear velocity; VSL, straight-line velocity; WOB, wobble coefficient (calculated as VAP/VCL x 100). ${ }^{a, b}$ Values within a row with different superscript letters indicates statistically significant differences.

in CTR, 5.4\% (10/186) in UND, and 13.5\% (16/118) in FA Group, respectively.

\section{Discussion}

Although maternal dietary restriction during early pregnancy did not result in any gross phenotypic difference in the progeny, including birth weight and growth rate up to 12 months of age, the data presented here show that nutritional differences cause epigenetic modifications in the spermatozoa of the offspring. This is consistent with studies that show nutritional stress in early pregnancy does not affect growth (Everitt 1964, Parr et al. 1986, Rae et al. 2002), whereas postnatal growth is compromised when nutritional restriction is applied from mid-gestation until term (Schinckel \& Short 1961, Robinson \& McDonald 1979), Ford et al. 2007. Semen collected from male progeny of the undernourished dams showed reduced total and progressive motility compared with controls. Likewise, there was a trend in higher abnormalities in the chromatin structure (ATSD value, see Table 3 ) in the progeny of nutritionally stressed dams, but was still below the subfertility threshold in

Table 4 IVF outcomes: IVF was performed using sperm from lambs born from control (CTR), undernourished (UND) and Folic Acid supplemented (FA) ewes. Development time points were 2 cells and blastocyst stages.

\begin{tabular}{lccl}
\hline & Oocytes $(n)$ & 2-Cells stage embryo $(\%)$ & Blastocysts $(\%)$ \\
\hline CTR & 178 & $56 / 178(31.5)$ & $26 / 178(14.6)$ \\
UND & 186 & $44 / 186(23.6)^{b}$ & $10 / 186(5.4)^{\mathrm{a}, \mathrm{b}}$ \\
FA & 118 & $46 / 118(39)$ & $16 / 118(13.5)$ \\
\hline
\end{tabular}

a $P<0.005$ between UND and CTR, ${ }^{b} P<0.05$ between UND and FA; Fisher's exact test. rams. The sperm chromatin parameters evaluated are correlated with DNA strand breaks (Sailer et al. 1995) and fertility (Evenson et al. 1980). A significant negative correlation between sperm chromatin index and classical semen quality parameters has been reported for rams (Peris et al. 2004) and others species including human (Evenson et al. 1999, Januskauska et al. 2001, Saleh et al. 2002).

We also found that the early development of embryos produced by in vitro fertilization and embryo culture using the spermatozoa of lambs born to feed restricted ewes was reduced compared with controls; an effect partially compensated by folic acid supplementation. The supplementation with folic acid may have partially corrected the effects of nutrient restriction, increasing the availability of methyl groups that are broadly required in this developmental window for the acquisition of paternal imprinted regions and transposon silencing in male gonocyte (Trasler 2009). Although the overall level of global methylation was not altered by maternal dietary restriction, specific loci did show differential methylation between UND, FA, and CTR groups. Studies on mice have shown that postnatal feed restriction alters methylation across the genome in an unpredictable way, an effect detectable over two successive generations (Xue et al. 2016), even though the affected loci differ in each generation. Undernourishment in utero, however, affects the germline DNA methylome of F1 adult males in a locus-specific manner, rather than a genome-wide manner (Martínez et al. 2014, Radford et al. 2014). In the current study, we identified changes in DNA methylation in genes essential for sperm maturation and function, including laminin A (LMNA) (Alsheimer et al. 2004, Shen et al. 2014) and $\beta$-Transducin repeat- 
containing protein ( $\beta$-TRCP2; FBXW11) (Nakagawa et al. 2017) between the UND vs CTR groups. These results suggest a direct effect of the maternal nutritional restriction on the offspring sperm methylome, as there were genes related to sperm specific function among the DMRs detected.

Further investigations are needed to understand if the epigenetic changes identified are responsible for the reduced sperm quality, which may have been related to the lower embryo developmental rate in the UND group. Interestingly, the in vitro development of oocytes fertilized with spermatozoa of the FA group was similar to the CTR one, although there were many differences in DMRs between the FA and CTR groups. The undernourished animals supplemented with FA showed sperm methylation alterations in specific genes involved in the regulation of PI3K activation during spermatozoa capacitation, such as protein kinase $\mathrm{C}$ alpha (PRKCA) (Rotman et al. 2010) or spermatozoa motility, such as parkin (PARK2) (Borowska et al. 2018). Recently, PARK2 was proposed as candidate gene associated with quality variation in bull semen, including total sperm motility, amplitude of lateral head displacement, mitochondrial integrity, and ATP content (Borowska et al. 2018). This could explain the lower levels of sperm motility found in the FA group, notwithstanding the better developmental potential of IVF embryos obtained from these samples. Differential cytosine methylation has also been reported in these genes in primordial germ cells (PGCs) of mouse concepti that underwent intrauterine hyperglycaemic exposure (Ren et al. 2018).

In conclusion, we report here that maternal undernutrition during the pre- to post-fertilization period results in an alteration of the methylation profile in the spermatozoa of the resulting offspring. Embryos produced in vitro using spermatozoa from the low nutrition group had a reduced developmental potential compared with the controls. However, sperm from lambs of ewes supplemented with folic acid produced embryos with increased developmental potential over the nutrition restricted group, despite there being DMR between the CTR and FA groups. The relationship between these DMRs and development is yet to be explored.

\section{Supplementary materials}

This is linked to the online version of the paper at https://doi. org/10.1530/REP-19-0549.

\section{Declaration of interest}

The authors declare that there is no conflict of interest that could be perceived as prejudicing the impartiality of the research reported.

\section{Funding}

The design of the study, animal management samples collection and data analysis were supported by GenHome project MIUR/CNR, Programma FIRB. GA n. B81J12002520001 'Technological Resort for the Advancement of Animal Genomic Research.' This project has received funding from the European Union's Horizon 2020 research and innovation programme under grant agreement No. 734434 and from programme Twinning action (No. 692185) for data analysis and manuscript preparation.

\section{Author contribution statement}

J L W, P A M and P L conceived and planned the experiments; $P \mathrm{~L}$ supervised the animal work and collected the samples; J L W, P A M and P L critically analysed the results and contributed to manuscript writing; $\mathrm{P} T$ contributed to sample collection, E C and P T ran experiments, B L, E C and P T analysed and interpreted the data; $\mathrm{P}$ T wrote the manuscript; A S and P A S provided advice; F P and F T performed in vitro semen analysis (CASA) and cytofluorimetric analysis; D A A, collected semen and performed in vitro fertilization studies. All authors read and approved the final manuscript.

\section{References}

Alsheimer M, Liebe B, Sewell L, Stewart CL, Scherthan H \& Benavente R 2004 Disruption of spermatogenesis in mice lacking A-type lamins. Journal of Cell Science 117 1173-1178. (https://doi.org/10.1242/ jcs.00975)

Anderson OS, Sant KE \& Dolinoy DC 2012 Nutrition and epigenetics: an interplay of dietary methyl donors, one-carbon metabolism and DNA methylation. Journal of Nutritional Biochemistry 23 853-859. (https:// doi.org/10.1016/j.jnutbio.2012.03.003)

Anzalone DA, luso D, Czernik M, Ptak G \& Loi P 2016 Plasma membrane and acrosome loss before ICSI is required for sheep embryonic development. Journal of Assisted Reproduction and Genetics 33 757-763. (https://doi.org/10.1007/s10815-016-0709-1)

Ashe A, Sapetschnig A, Weick EM, Mitchell J, Bagijn MP, Cording AC, Doebley AL, Goldstein LD, Lehrbach NJ, Le Pen J et al. 2012 piRNAs can trigger a multigenerational epigenetic memory in the germline of C. elegans. Cell 150 88-99. (https://doi.org/10.1016/j. cell.2012.06.018)

Barker DJ, Gluckman PD, Godfrey KM, Harding JE, Owens JA \& Robinson JS 1993 Fetal nutrition and cardiovascular disease in adult life. Lancet 341 938-941. (https://doi.org/10.1016/0140-6736(93)91224-a)

Bernal AJ \& Jirtle RL 2010 Epigenomic disruption: the effects of early developmental exposures. Birth Defects Research: Part A, Clinical and Molecular Teratology 88 938-944. (https://doi.org/10.1002/ bdra.20685)

Bindea G, Mlecnik B, HackI H, Charoentong P, Tosolini M, Kirilovsky A, Fridman WH, Pagès F, Trajanoski Z \& Galon J 2009 ClueGO: a cytoscape plug-in to decipher functionally grouped gene ontology and pathway annotation networks. Bioinformatics 25 1091-1093. (https:// doi.org/10.1093/bioinformatics/btp101)

Borowska A, Szwaczkowski T, Kamiński S, Hering DM, Kordan W \& Lecewicz M 2018 Identification of genome regions determining semen quality in Holstein-Friesian bulls using information theory. Animal Reproduction Science 192 206-215. (https://doi.org/10.1016/j. anireprosci.2018.03.012)

Butler H \& Juurlink BHJ 1987 An Atlas for Staging Mammalian and Chick Embryos, p. 218. Boca Raton Fla: CRC Press, Taylor \& Francis Group.

Capra E, Turri F, Lazzari B, Cremonesi P, Gliozzi TM, Fojadelli I, Stella A \& Pizzi F 2017 Small RNA sequencing of cryopreserved semen from single bull revealed altered miRNAs and piRNAs expression between 
high- and low-motile sperm populations. BMC Genomics 18 14. (https:// doi.org/10.1186/s12864-016-3394-7)

Cetin I, Berti C \& Calabrese S 2010 Role of micronutrients in the periconceptional period. Human Reproduction Update 16 80-95. (https://doi.org/10.1093/humupd/dmp025)

Daxinger L \& Whitelaw E 2012 Understanding transgenerational epigenetic inheritance via the gametes in mammals. Nature Reviews: Genetics 13 153-162. (https://doi.org/10.1038/nrg3188)

Evenson D \& Jost L 2000 Sperm chromatin structure assay is useful for fertility assessment. Methods in Cell Science 22 169-189. (https://doi. org/10.1023/a:1009844109023)

Evenson DP, Darzynkiewicz Z \& Melamed MR 1980 Relation of mammalian sperm chromatin heterogeneity to fertility. Science $\mathbf{2 1 0}$ 1131-1133. (https://doi.org/10.1126/science.7444440)

Evenson DP, Jost LK, Marshall D, Zinaman MJ, Clegg E, Purvis K, de Angelis P \& Claussen OP 1999 Utility of the sperm chromatin structure assay as a diagnostic and prognostic tool in the human fertility clinic. Human Reproduction 14 1039-1049. (https://doi.org/10.1093/ humrep/14.4.1039)

Everitt GC 1964 Maternal undernutrition and retarded fetal development in merino sheep. Nature 201 1341-1342. (https://doi. org/10.1038/2011341a0)

Faulk C \& Dolinoy DC 2013 Complex phenotypes: epigenetic manifestation of environmental exposures. In Environmental Epigenomics in Health and Disease. Eds RL Jirtle \& FL Tyson. Springer.

Ford SP, Hess BW, Schwope MM, Nijland MJ, Gilbert JS, Vonnahme KA, Means WJ, Han H \& Nathanielsz PW 2007 Maternal undernutrition during early to mid-gestation in the ewe results in altered growth, adiposity, and glucose tolerance in male offspring. Journal of Animal Science 85 1285-1294. (https://doi.org/10.2527/jas.2005-624)

Gliozzi TM, Turri F, Manes S, Cassinelli C \& Pizzi F 2017 The combination of kinetic and flow cytometric semen parameters as a tool to predict fertility in cryopreserved bull semen. Animal 11 1975-1982. (https://doi. org/10.1017/S1751731117000684)

Gluckman PD, Hanson MA \& Beedle AS 2007 Non-genomic transgenerational inheritance of disease risk. BioEssays 29 145-154. (https://doi.org/10.1002/bies.20522)

Greer EL, Maures TJ, Hauswirth AG, Green EM, Leeman DS, Maro GS, Han S, Banko MR, Gozani O \& Brunet A 2010 Members of the H3K4 trimethylation complex regulate lifespan in a germline-dependent manner in C. elegans. Nature 466 383-387. (https://doi.org/10.1038/ nature09195)

Hackett JA \& Surani MA 2013 DNA methylation dynamics during the mammalian life cycle. Philosophical Transactions of the Royal Society of London: Series B, Biological Sciences 368 20110328. (https://doi. org/10.1098/rstb.2011.0328)

Hill PWS, Leitch HG, Requena CE, Sun Z, Amouroux R, Roman-Trufero M, Borkowska M, Terragni J, Vaisvila R, Linnett S et al. 2018 Epigenetic reprogramming enables the transition from primordial germ cell to gonocyte. Nature 555 392-396. (https://doi.org/10.1038/nature25964)

Huypens P, Sass S, Wu M, Dyckhoff D, Tschöp M, Theis F, Marschall S, Hrabě de Angelis M \& Beckers J 2016 Epigenetic germline inheritance of diet-induced obesity and insulin resistance. Nature Genetics $\mathbf{4 8}$ 497-499. (https://doi.org/10.1038/ng.3527)

Januskauska A, Johannisson A \& Rodriguez-Martinez H 2001 Assessment of sperm quality through fluorometry and sperm chromatin structure assay in relation to field fertility of frozen-thawed semen from Swedish Al bulls. Theriogenology 55 947-961. (https://doi.org/10.1016/s0093691x(01)00456-3)

Langley SC \& Jackson AA 1994 Increased systolic blood pressure in adult rats induced by fetal exposure to maternal low protein diets. Clinical Science 86 217-222; discussion 121. (https://doi.org/10.1042/ cs0860217)

Langley-Evans SC, Gardner DS \& Welham SJ 1998 Intrauterine programming of cardiovascular disease by maternal nutritional status. Nutrition 14 39-47. (https://doi.org/10.1016/s0899-9007(97)00391-2)

Ledda S, Bogliolo L, Bebbere D, Ariu F \& Pirino S 2010 Characterization, isolation and culture of primordial germ cells in domestic animals: recent progress and insights from the ovine species. Theriogenology 74 534-543. (https://doi.org/10.1016/j.theriogenology.2010.05.011)

Martínez D, Pentinat T, Ribó S, Daviaud C, Bloks VW, Cebrià J, Villalmanzo N, Kalko SG, Ramón-Krauel M, Díaz R et al. 2014 In utero undernutrition in male mice programs liver lipid metabolism in the second-generation offspring involving altered LXRa DNA methylation. Cell Metabolism 19 941-951. (https://doi.org/10.1016/j. cmet.2014.03.026)

Messerschmidt DM, Knowles BB \& Solter D 2014 DNA methylation dynamics during epigenetic reprogramming in the germline and preimplantation embryos. Genes and Development 28 812-828. (https://doi.org/10.1101/gad.234294.113)

Nakagawa T, Zhang T, Kushi R, Nakano S, Endo T, Nakagawa M, Yanagihara N, Zarkower D \& Nakayama K 2017 Regulation of mitosismeiosis transition by the ubiquitin ligase $\beta-\operatorname{TrCP}$ in male germ cells. Development 144 4137-4147. (https://doi.org/10.1242/dev.158485)

Parr RA, Williams AH, Campbell IP, Witcombe GF \& Roberts AM 1986 Low nutrition of ewes in early pregnancy and the residual effect on the offspring. Journal of Agricultural Science 106 81-87. (https://doi. org/10.1017/S0021859600061773)

Patti ME 2013 Intergenerational programming of metabolic disease: evidence from human populations and experimental animal models. Cellular and Molecular Life Sciences 70 1597-1608. (https://doi. org/10.1007/s00018-013-1298-0)

Peris SI, Morrier A, Dufour M \& Bailey JL 2004 Cryopreservation of ram semen facilitates sperm DNA damage: relationship between sperm andrological parameters and the sperm chromatin structure assay. Journal of Andrology 25 224-233. (https://doi.org/10.1002/j.1939-4640.2004. tb02782.x)

Radford EJ, Ito $M$, Shi $H$, Corish JA, Yamazawa K, Isganaitis E, Seisenberger S, Hore TA, Reik W, Erkek S et al. 2014 In utero effects. In utero undernourishment perturbs the adult sperm methylome and intergenerational metabolism. Science 345 1255903. (https://doi. org/10.1126/science.1255903)

Rae MT, Kyle CE, Miller DW, Hammond AJ, Brooks AN \& Rhind SM 2002 The effects of undernutrition, in utero, on reproductive function in adult male and female sheep. Animal Reproduction Science 72 63-71. (https://doi.org/10.1016/s0378-4320(02)00068-4)

Rando OJ 2012 Daddy issues: Paternal effects on pheno- type. Cell $\mathbf{1 5 1}$ 702-708. (https://doi.org/10.1016/j.cell.2012.10.020)

Ren J, Cheng Y, Ming ZH, Dong XY, Zhou YZ, Ding GL, Pang HY, Rahman TU, Akbar R, Huang HF et al. 2018 Intrauterine hyperglycemia exposure results in intergenerational inheritance via DNA methylation reprogramming on F1 PGCs. Epigenetics and Chromatin 11 20. (https:// doi.org/10.1186/s13072-018-0192-2)

Robinson JJ \& McDonald I 1979 Ovine prenatal growth, its mathematical description and the effects of maternal nutrition. Annales de Biologie Animale Biochimie Biophysique 19 225-234. (https://doi.org/10.1051/ rnd:19790210)

Rodgers AB, Morgan CP, Leu NA \& Bale TL 2015 Transgenerational epigenetic programming via sperm microRNA recapitulates effects of paternal stress. PNAS 112 13699-13704. (https://doi.org/10.1073/ pnas.1508347112)

Rotman T, Etkovitz N, Spiegel A, Rubinstein S \& Breitbart H 2010 Protein kinase $\mathrm{A}$ and protein kinase C(alpha)/PPP1CC2 play opposing roles in the regulation of phosphatidylinositol 3-kinase activation in bovine sperm. Reproduction 140 43-56. (https://doi.org/10.1530/REP-090314)

Sailer BL, Jost LK \& Evenson DP 1995 Mammalian sperm DNA susceptibility to in situ denaturation associated with the presence of DNA strand breaks as measured by the terminal deoxynucleotidyl transferase assay. Journal of Andrology 16 80-87. (https://doi.org/10.1002/j.1939-4640.1995. tb01735.x)

Saleh RA, Agarwal A, Nelson DR, Nada EA, El-Tonsy MH, Alvarez JG, Thomas Jr AJ \& Sharma RK 2002 Increased sperm nuclear DNA damage in normozoospermic infertile men: a prospective study. Fertility and Sterility 78 313-318. (https://doi.org/10.1016/s0015-0282(02)03219-3)

Schinckel PG \& Short BF 1961 Influence of nutritional level during prenatal and early post-natal life on adult fleece and body characters. Australian Journal of Agricultural Research 12 176-202. (https://doi. org/10.1071/AR9610176)

Seisenberger S, Peat JR, Hore TA, Santos F, Dean W \& Reik W 2013 Reprogramming DNA methylation in the mammalian life cycle: building and breaking epigenetic barriers. Philosophical Transactions of the Royal Society of London: Series B, Biological Sciences 368 20110330. (https:// doi.org/10.1098/rstb.2011.0330) 
Seong KH, Li D, Shimizu H, Nakamura R \& Ishii S 2011 Inheritance of stress-induced, ATF-2-dependent epigenetic change. Cell 145 1049-1061. (https://doi.org/10.1016/j.cell.2011.05.029)

Shen J, Chen W, Shao B, Qi Y, Xia Z, Wang F, Wang L, Guo X, Huang X \& Sha J 2014 Lamin A/C proteins in the spermatid acroplaxome are essential in mouse spermiogenesis. Reproduction 148 479-487. (https:// doi.org/10.1530/REP-14-0012)

Siklenka K, Erkek S, Godmann M, Lambrot R, McGraw S, Lafleur C, Cohen T, Xia J, Suderman M, Hallett M et al. 2015 Disruption of histone methylation in developing sperm impairs offspring health transgenerationally. Science $\mathbf{3 5 0}$ aab2006. (https://doi.org/10.1126/ science.aab2006)

Sinclair KD, Karamitri A \& Gardner DS 2010 Dietary regulation of developmental programming in ruminants: epigenetic modifications in the germline. Society of Reproduction and Fertility Supplement 67 59-72. (https://doi.org/10.5661/RDR-VII-59)

Sturn A, Quackenbush J \& Trajanoski Z 2002 Genesis: cluster analysis of microarray data. Bioinformatics 18 207-208. (https://doi.org/10.1093/ bioinformatics/18.1.207)

Trasler JM 2009 Epigenetics in spermatogenesis. Molecular and Cellular Endocrinology 306 33-36. (https://doi.org/10.1016/j.mce.2008.12.018)

Van Soom A, Vandaele L, Goossens K, Heras S, Wydooghe E, Rahman MB, Kamal MM, Van Eetvelde M, Opsomer G \& Peelman L 2013 Epigenetics and the periconception environment in ruminants. Proceeding of the Belgian Royal Academies of Medicine 2 1-23.
Wei Y, Schatten H \& Sun QY 2015 Environmental epigenetic inheritance through gametes and implications for human reproduction. Human Reproduction Update 21 194-208. (https://doi.org/10.1093/humupd/ dmu061)

Xue J, Schoenrock SA, Valdar W, Tarantino LM \& Ideraabdullah FY 2016 Maternal vitamin D depletion alters DNA methylation at imprinted loci in multiple generations. Clinical Epigenetics 8 107. (https://doi. org/10.1186/s13148-016-0276-4)

Zambrano E, Rodrigez-González GL, Guzmán C, García-Becerra R, Boeck L, Díaz L, Menjivar M, Larrea F \& Nathanielsz PW 2005 A maternal low protein diet during pregnancy and lactation in the rat impairs male reproductive development. Journal of Physiology $\mathbf{5 6 3}$ 275-284. (https://doi.org/10.1113/jphysiol.2004.078543)

Ziller MJ, Hansen KD, Meissner A \& Aryee MJ 2015 Coverage recommendations for methylation analysis by whole-genome bisulfite sequencing. Nature Methods 12 230-232. (https://doi.org/10.1038/ nmeth.3152)

Received 13 November 2019

First decision 9 December 2019

Revised manuscript received 8 January 2020

Accepted 6 February 2020 